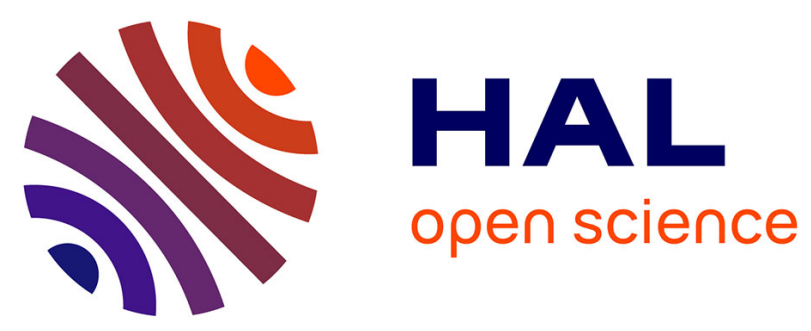

\title{
Extended-Braking-Stiffness Estimation Under Varying Road-Adherence Conditions
}

\author{
Missie Aguado-Rojas, William Pasillas-Lépine, Antonio Loria
}

\section{To cite this version:}

Missie Aguado-Rojas, William Pasillas-Lépine, Antonio Loria. Extended-Braking-Stiffness Estimation Under Varying Road-Adherence Conditions. IEEE Transactions on Control Systems Technology, 2020, 28 (5), pp.1964-1971. 10.1109/TCST.2019.2918483 . hal-02367531

\section{HAL Id: hal-02367531 \\ https://hal.science/hal-02367531}

Submitted on 5 Mar 2020

HAL is a multi-disciplinary open access archive for the deposit and dissemination of scientific research documents, whether they are published or not. The documents may come from teaching and research institutions in France or abroad, or from public or private research centers.
L'archive ouverte pluridisciplinaire HAL, est destinée au dépôt et à la diffusion de documents scientifiques de niveau recherche, publiés ou non, émanant des établissements d'enseignement et de recherche français ou étrangers, des laboratoires publics ou privés. 


\title{
Extended-braking-stiffness estimation under varying road-adherence conditions
}

\author{
Missie Aguado-Rojas, William Pasillas-Lépine, Antonio Loría
}

\begin{abstract}
We present a switched adaptive observer for the estimation of the tyre extended braking stiffness (XBS) during ABS braking scenarios. The design of the observer is based on Burckhardt's friction model, and on a model of the wheel dynamics in which the XBS appears as one of the state variables. Global asymptotic stability of the estimation error is established provided that certain dwell-time and persistency of excitation conditions hold. The approach is validated under constant road conditions with experimental data from a tyre-in-the-loop test rig, and under varying road conditions via numerical simulations on a two-axle vehicle model that includes dynamic load transfer and tyre relaxation length.
\end{abstract}

Index Terms-adaptive observer, switched systems, antilock braking system, persistency of excitation, time-scale transformation, non-strict Lyapunov functions, tyre-road friction.

\section{INTRODUCTION}

As vehicle safety-oriented control systems become more advanced, their dependence on accurate information on the state of the vehicle and its surroundings increases. For instance, the performance of driver-assistance technologies, such as the antilock braking system (ABS), is greatly influenced by the characteristics of the friction force between the tyre and the road. Therefore, by taking into account the external driving conditions of the vehicle, the effectiveness of such active safety systems can be greatly improved [1]. Tyre-road friction, however, cannot be directly measured in real-time; hence its estimation has been an intensive research area in the last years. Numerous different approaches to estimate the tyre-road friction coefficient and its maximum value have been proposed in the literature — see, e.g., [2], [3], and references therein. In several of these works it is proposed to estimate the peak tyre-road friction under the premise that the tyre braking stiffness indicates the peak value of the friction-slip curve [4]. The braking stiffness is the slope of the friction with respect to the wheel slip at the zero-friction operating point [5]. In this work we are interested in a generalization of this concept that is known as extended braking stiffness (XBS) and may be defined as the slope of the friction-slip curve at any operating point — see [6] and [7]. The interest of estimating the XBS is that, in contrast to the unknown optimal value of wheel slip,

Research funded in part by ANR via project HANDY, number ANR18-CE40-0010. The work of M. Aguado-Rojas was supported by Consejo Nacional de Ciencia y Tecnología and Secretaría de Educación Pública, Mexico.

M. Aguado-Rojas is with L2S-CentraleSupélec, Université ParisSud, Université Paris-Saclay, 91192 Gif-sur-Yvette, France (e-mail: missie.aguado@12s.centralesupelec.fr).

W. Pasillas-Lépine and A. Loría are with L2S-CentraleSupélec, CNRS, 91192 Gif-sur-Yvette, France (e-mail: pasillas@12s.centralesupelec.fr; loria@12s.centralesupelec.fr). the optimal value of XBS is always known and equal to zero. Hence, an ABS control algorithm can be designed in order to maintain the XBS in a neighbourhood around zero.

The concept of maximizing the braking force in an ABS based on the slope of the braking force with respect to the wheel slip was first introduced by Sugai et al. in [6]. The $\mathrm{XBS}$ is identified by forcing a vibration via the brake actuator and analyzing the frequency characteristics of a resonance system composed of the vehicle body, the wheel and the road surface. In [7] the XBS is estimated via the instrumental variable method based on the frequency characteristics of a tyre vibration model linearized around a constant-velocity operating point. In [8], the XBS is (implicitly) assumed to be a constant parameter and estimated by applying the recursive least squares algorithm to wheel rotational velocities. In [9], in order to estimate the maximum friction coefficient, the XBS is used to signal the entrance of the tyre into a different road surface and to distinguish one type of road from another. The XBS is estimated using elementary diagnostics tools and algebraic methods to filter and estimate derivatives of noisy signals (whose main difficulty is to achieve a good trade-off between filtering and reactivity), and the estimation results are accurate only within certain validity range. In [10], Hoàng et al. introduced two different models of the wheel acceleration dynamics in which the XBS appears as one of the state variables. The first one is based on the well-known Burckhardt's friction model [11] and it allows to estimate the XBS under the assumption that the road parameters are partially known. To cope with uncertainty on all the road parameters, the second model is based on a re-parametrization of Burckhardt's model that is linear in the (new) unknown parameters. Assumed to be constant, these are regarded as static state variables and the XBS is estimated using an asymptotic state observer for the augmented system. The implementation of this observer, however, still requires knowledge on upper and lower bounds of the road parameters. As a consequence, the observer is not robust to large changes in road conditions, as conservative bounds must be chosen in order to take into account different types of roads.

In this paper we present a switched adaptive observer to estimate the XBS using the first model of [10]. Our approach, however, does not require any a priori knowledge on the parameters related to the road conditions or their bounds. Instead of changing the friction model, a state-space transformation is used to preserve Burckhardt's standard parametrization and render the system linear in the unknown variables. The proposed observer has an overall better performance than that of [10] and provides a good estimation of the XBS even after 
large changes in road conditions. This paper builds upon the preliminary results presented in [12], which are extended here in three directions. First, the stability properties of the observer are established with a more rigorous theoretical analysis and clearer hypotheses. Conditions which might hamper the usefulness of the approach in particular control tasks are discussed as well. Second, the observer is experimentally tested under constant road conditions and vertical load, characteristics which are imposed by the test rig. Third, the observer is tested in simulation under varying road conditions with a vehicle model that includes dynamic load transfer and tyre relaxation length.

\section{XBS DYNAMICS}

The model employed in this paper to describe the XBS dynamics is based on a simplified single-wheel model that considers the forces acting on the longitudinal direction only. Despite its simplicity, the latter is widely encountered in the literature of active braking control systems, as it is known to provide a simple yet sufficiently rich description of the braking dynamics (see, e.g., [13, Ch. 2]).

The dynamics of the angular velocity $\omega$ of the wheel is described by

$$
I \dot{\omega}=-R F_{x}+T,
$$

where $I$ is the rotational inertia of the wheel, $R$ is its effective rolling radius, $F_{x}$ is the longitudinal tyre force, and $T=T_{e}-T_{b}$ is the torque applied to the wheel, composed by the engine torque $T_{e}$ and the brake torque $T_{b}$. In what follows it is assumed that the clutch is open during an ABS braking maneuver so the engine torque can be neglected. The brake torque is given by $T_{b}=\gamma_{b} P_{b}$, where $\gamma_{b}$ denotes the brake efficiency, and $P_{b}$ denotes the brake pressure.

The longitudinal tyre force $F_{x}$ is modeled as

$$
F_{x}=\mu(\lambda) F_{z}
$$

where $F_{z}$ is the tyre normal load and $\mu(\lambda)$ denotes the tyreroad friction (or adhesion) coefficient, which describes the tyre capability of transferring the vertical load to the ground. It depends nonlinearly on the longitudinal wheel slip $\lambda$ defined as

$$
\lambda=\frac{R \omega-v_{x}}{v_{x}},
$$

where $v_{x}$ is the longitudinal speed of the vehicle and $R \omega$ is the linear speed of the tyre at the wheel-ground contact point. From physical considerations, in what follows $v_{x}$ is assumed to be positive, bounded, and separated from zero, i.e.,

$$
v_{x \min } \leq v_{x}(t) \leq v_{x \max }, \quad \forall t \geq 0 .
$$

The first inequality above is justified by the fact that, according to certain manufacturers, the ABS is automatically deactivated when the speed is lower than $5 \mathrm{~km} / \mathrm{h}$.

The friction coefficient is described using Burckhardt's model

$$
\mu(\lambda)=c_{1}\left(1-\exp \left(-c_{2} \lambda\right)\right)-c_{3} \lambda,
$$

where the coefficients $c_{i}$ are "constants" that depend on the road conditions, on the tyre characteristics, and on the vehicle

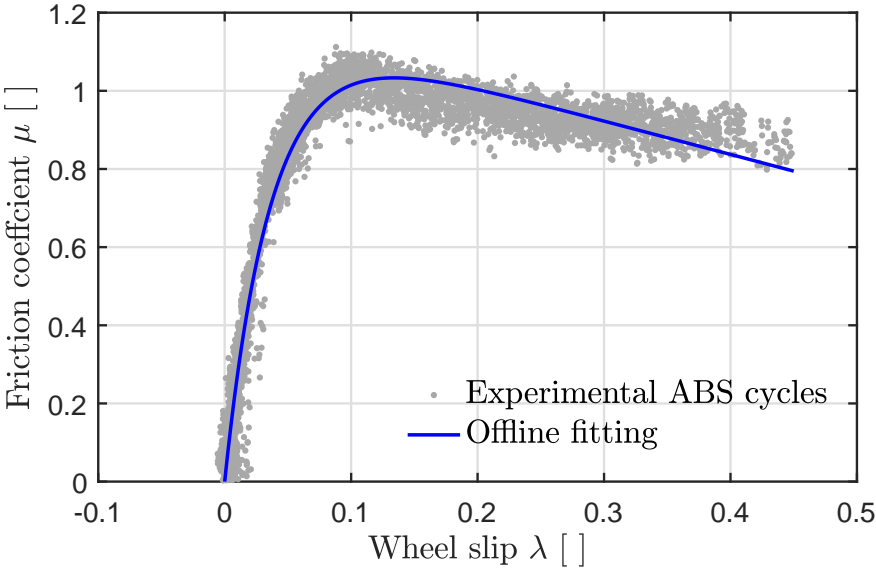

Fig. 1. Measurement and offline fitting of the friction characteristics of the tyre-in-the-loop test rig. The parameters associated to the tyre mounted on the rim are $I=1.2 \mathrm{~kg} \cdot \mathrm{m}^{2}, R=0.3 \mathrm{~m}, F_{z}=2850 \mathrm{~N} \cdot \mathrm{m}$, and $\gamma_{b}=17.5$ $\mathrm{N} \cdot \mathrm{m} / \mathrm{bar}$. The fitted values of Burckhardt's friction model are $c_{1}=1.1794$, $c_{2}=27$, and $c_{3}=0.8552$. For the half of the curve with negative $\lambda$, the parameters $c_{1}$ and $c_{2}$ are also negative.

operational conditions [11]. Thus, by changing the values of these three parameters, many different conditions can be modeled. The typical behavior of the friction coefficient is illustrated in Fig. 1. The dots correspond to experimental data obtained from a tyre-in-the-loop test rig, while the solid line corresponds to the offline fitting of the data to the model (5). The XBS, which is the variable of interest in this paper, is defined as the slope of this curve at the operating point, i.e.,

$$
\mu^{\prime}(\lambda)=\frac{d \mu}{d \lambda}(\lambda)
$$

It indicates whether the current slip is close to the maximum force. In contrast to the maximum friction coefficient, which depends on the road conditions and is usually unknown, the XBS that corresponds to the optimal wheel slip is always known and equal to zero.

We define as state variables the wheel acceleration offset

$$
z_{1}:=R \dot{\omega}-a_{x}(t),
$$

i.e., the difference between the longitudinal acceleration $a_{x}(t)$ of the vehicle and the linear acceleration of the tyre at the wheel-ground contact point, and the XBS $z_{2}:=\mu^{\prime}(\lambda)$. During an ABS braking maneuver, the longitudinal acceleration of the vehicle remains almost constant and close to the maximal value allowed by the road conditions, while the wheel slip remains relatively small. Under such conditions, the dynamics of $z_{1}$ and $z_{2}$ are described by ${ }^{1}$

$$
\begin{aligned}
& \dot{z}_{1}=-\frac{a}{v_{x}(t)} z_{1} z_{2}-b u \\
& \dot{z}_{2}=\left(c z_{2}+d\right) \frac{1}{v_{x}(t)} z_{1},
\end{aligned}
$$

where the control input $u=\dot{P}_{b}$ is the derivative of the brake pressure, $a=\left(R^{2} / I\right) F_{z}$ and $b=(R / I) \gamma_{b}$ are assumed to be known constant parameters, and $c=-c_{2}$ and $d=-c_{2} c_{3}$ are

\footnotetext{
${ }^{1}$ The reader is referred to [10], as well as [12], for the complete derivation of this simplified model.
} 
parameters that depend on the road conditions, thus they are assumed to be constant but unknown. Moreover, it is assumed that $u$ is bounded and it is such that the state trajectories of (7) are bounded. The vehicle speed $v_{x}(t)$ is considered as a known external variable. The wheel acceleration offset defined in (6) can be computed from the measurements of the wheel angular acceleration and the vehicle longitudinal acceleration. Hence, $z_{1}$ is regarded as the system's measured output. Our aim in this paper is to design an observer for the unmeasurable XBS $z_{2}$.

\section{SWITCHED ADAPTIVE OBSERVER}

In this section we address the problem of XBS estimation during an ABS braking maneuver under the conditions previously described.

\section{A. Observer design}

The system (7) is linear in the unmeasured state $z_{2}$; hence, at first sight, one could rely on theory of observer design for such systems — see e.g., [14], [15], as well as [16]-[18]. However, since $c$ and $d$ are assumed to be unknown, the term $c z_{2}$ is nonlinear in two of the unknown variables. To cope with this difficulty, we introduce the linear change of coordinates

$$
\begin{aligned}
& w_{1}=z_{1} \\
& w_{2}=z_{2}+\frac{c}{a} z_{1}
\end{aligned}
$$

that transforms the system (7) into

$$
\begin{aligned}
& \dot{w}_{1}=\frac{w_{1}}{v_{x}(t)}\left(c w_{1}-a w_{2}\right)-b u \\
& \dot{w}_{2}=-\frac{b c}{a} u+\frac{w_{1}}{v_{x}(t)} d .
\end{aligned}
$$

In the new coordinates, the previous equations become

$$
\begin{aligned}
\dot{w} & =A(t, y) w+B u+\Psi(t, u, y) \theta \\
y & =C w,
\end{aligned}
$$

where $w=\left[\begin{array}{ll}w_{1} & w_{2}\end{array}\right]^{\top}$,

$$
A(t, y)=\frac{y}{v_{x}(t)}\left[\begin{array}{cc}
0 & -a \\
0 & 0
\end{array}\right]:=\frac{y}{v_{x}(t)} A^{\prime}
$$

$B=\left[\begin{array}{c}-b \\ 0\end{array}\right], \quad C=\left[\begin{array}{ll}1 & 0\end{array}\right], \quad \Psi(t, u, y)=\left[\begin{array}{cc}\frac{y^{2}}{v_{x}(t)} & 0 \\ -\frac{b}{a} u & \frac{y}{v_{x}(t)}\end{array}\right]$,

$\theta=\left[\begin{array}{ll}c & d\end{array}\right]^{\top}$, and the measured output $y=w_{1}$ is the wheel acceleration offset previously defined in (6).

Then, inspired by [16], we introduce the adaptive observer

$$
\begin{aligned}
\dot{\hat{w}}= & A(t, y) \hat{w}+B u+\Psi(t, u, y) \hat{\theta} \\
& +\left[K(t, y)+\Upsilon \Gamma \Upsilon^{\top} C^{\top}\right](y-C \hat{w}) \\
\dot{\hat{\theta}}= & \Gamma \Upsilon^{\top} C^{\top}(y-C \hat{w}), \\
\dot{\Upsilon}= & {[A(t, y)-K(t, y) C] \Upsilon+\Psi(t, u, y) }
\end{aligned}
$$

where $\Gamma=\Gamma^{\top}>0$. This observer is also reminiscent of that in [15, Def. 5.3.2] where $A$ is considered to be constant. A benefit of this observer is that the dynamics of the estimation errors $\tilde{w}:=\hat{w}-w$ and $\tilde{\theta}:=\hat{\theta}-\theta$ corresponds to

$$
\begin{aligned}
\dot{\tilde{w}}= & {[A(t, y)-K(t, y) C] \tilde{w}+\Psi(t, u, y) \tilde{\theta} } \\
& -\Upsilon \Gamma \Upsilon^{\top} C^{\top} C \tilde{w} \\
\dot{\tilde{\theta}}= & -\Gamma \Upsilon^{\top} C^{\top} C \tilde{w}
\end{aligned}
$$

together with (10c). For (11), it is shown in [16] that the origin is (uniformly) asymptotically stable ${ }^{2}$ if $K(t, y)$ is such that the origin for the system

$$
\dot{\tilde{w}}=(A(t, y)-K(t, y) C) \tilde{w}
$$

is also (uniformly) asymptotically stable and $\Psi(t, u, y)$ is persistently exciting, i.e., if there exist $\mu_{1}>0$ and $T_{1}>0$ such that, for all $t \geq 0$,

$$
\int_{t}^{t+T_{1}} \Psi(\varsigma, u(\varsigma), y(\varsigma))^{\top} \Psi(\varsigma, u(\varsigma), y(\varsigma)) d \varsigma \geq \mu_{1} \mathbf{I} .
$$

Thus, if $\hat{w} \rightarrow w$ and $\hat{\theta} \rightarrow \theta$, the estimate of the XBS, which is computed via the inverse change of coordinates

$$
\begin{aligned}
& \hat{z}_{1}=\hat{w}_{1} \\
& \hat{z}_{2}=\hat{w}_{2}-\frac{\hat{c}}{a} \hat{w}_{1},
\end{aligned}
$$

tends to its true values, i.e., $\hat{z} \rightarrow z$.

In what follows, we show how to define the observer gain $K(t, y)$ and establish global asymptotic stability of the origin for the system (12).

\section{B. Analysis under known road conditions}

For clarity of exposition, let us assume momentarily that $\hat{\theta}=\theta$ and $\Gamma=0$. In view of the structure of $A(t, y)$ in (9), we set

$$
K(t, y):=\frac{y}{v_{x}(t)} K^{\prime}(y)
$$

to obtain

$$
\dot{\tilde{w}}=\frac{y}{v_{x}(t)}\left[A^{\prime}-K^{\prime}(y) C\right] \tilde{w}
$$

where $K^{\prime}$ is yet to be defined. The state variable of this system is $\tilde{w}$. Hence, for the purpose of analysis, we write (14) as a linear time-varying system by replacing $y$ with the output trajectories $y(t)$, i.e.,

$$
\dot{\tilde{w}}=\frac{y(t)}{v_{x}(t)}\left[A^{\prime}-K^{\prime}(y(t)) C\right] \tilde{w} .
$$

Provided that $y(t)$ is defined for all $t \geq 0$, this system is well posed since $v_{x}(t)$ is bounded and separated from zero - cf. [19], [20, p. 627].

Let us assume momentarily that $y(t) \neq 0$. Multiplying both sides of (15) by $v_{x}(t) /|y(t)|$ we obtain

$$
\frac{v_{x}(t)}{|y(t)|} \frac{d \tilde{w}}{d t}=\operatorname{sgn}(y(t))\left[A^{\prime}-K^{\prime}(y(t)) C\right] \tilde{w}
$$

${ }^{2}$ Strictly speaking, in [16] the functions $A$ and $K$ only depend on $t$; hence exponential stability is established. Such property, however, is out of reach for (11). 
and it becomes natural to introduce the new time variable $\tau=$ $\nu(t)$, where

$$
\nu(t):=\int_{0}^{t} \frac{|y(\varsigma)|}{v_{x}(\varsigma)} d \varsigma .
$$

Although, at a first glance, it may appear erroneous to define a state-dependent time variable, this approach is not without precedent in the literature. It is inspired from the concept of orbital equivalence, which was popularized during the sixties and seventies as a tool for analyzing the qualitative properties of differential equations [21, p. 19]. Since then, it has been used to design control laws [22], adaptive filters [23], and observers [24], [25]. It is important to stress that the proposed change of time variable is not needed to implement the observer (10), but is only an artifice used in order to analyze the error dynamics. Therefore, whether the time scale depends on a state trajectory that is measurable or not is not a problem, as long as it is well defined (a property that will be guaranteed by Assumption 1 - see [26] for a detailed analysis of this last point). Hence, using

$$
\frac{d \tau}{d t}=\frac{|y(t)|}{v_{x}(t)}, \quad \tau(0)=0,
$$

and $t=\nu^{-1}(\tau)$, we may rewrite (16) as

$$
\frac{d \tilde{w}}{d \tau}=\mathcal{A}(\tau) \tilde{w}
$$

where $\mathcal{A}(\tau)=\operatorname{sgn}\left(y\left(\nu^{-1}(\tau)\right)\right)\left[A^{\prime}-K^{\prime}\left(y\left(\nu^{-1}(\tau)\right) C\right] \tilde{w}\right.$. Note that $\operatorname{sgn}\left(y\left(\nu^{-1}(\tau)\right)\right)=: \sigma(\tau)$ takes values in $\{-1,1\}$; hence, it may be regarded as a switching signal. In that light, it is also natural to define the control gain $K^{\prime}$ as

$$
K^{\prime}(y)= \begin{cases}{\left[\begin{array}{l}
k_{1}^{+} \\
k_{2}^{+}
\end{array}\right],} & \text {if } y>0 \\
{\left[\begin{array}{l}
k_{1}^{-} \\
k_{2}^{-}
\end{array}\right],} & \text {if } y<0\end{cases}
$$

where $k_{1,2}^{ \pm}$are parameters to be defined so that (19) become an exponentially-stable switched system defined by

$$
\frac{d \tilde{w}}{d \tau}=A_{\sigma(\tau)} \tilde{w}
$$

where

$$
A_{\sigma(\tau)}= \begin{cases}A_{+}=\left[\begin{array}{cc}
-k_{1}^{+} & -a \\
-k_{2}^{+} & 0
\end{array}\right], & \text { if } y(\tau)>0 \\
A_{-}=\left[\begin{array}{ll}
k_{1}^{-} & a \\
k_{2}^{-} & 0
\end{array}\right], & \text { if } y(\tau)<0 .\end{cases}
$$

Stability of switched systems is a well-studied subject [27]; sufficient conditions for this system to be exponentially stable are that $\sigma(\tau)$ admits a dwell time and that the dynamics of the system for each value of $\sigma$ is exponentially stable. Nevertheless, for standard theory on switched systems to apply to the system (21), several technical aspects must be taken into account. So far, we have assumed that the output trajectory $y(t) \neq 0$; this is needed for (16) to be well defined. Furthermore, we have implicitly assumed that the time-scale defined in (17) is well-posed.
Indeed, for this transformation to be well defined, the time $\tau$ must never evolve backwards relative to the actual time $t$, it must not go to infinity in finite time $t$, and it must not have a finite limit. The first condition holds because $\nu(t)$ is nondecreasing — see (18) and (4). The second condition is guaranteed by the fact that $y$ is bounded and the vehicle's velocity satisfies (4) - see (17). To guarantee the third condition we rely on typical wheel acceleration profiles that correspond to the operation of commercial ABS — see, e.g., [28, pp. 82-89] and $[29, \S 30.1 .3]$.

Assumption 1. During an ABS braking maneuver the wheel acceleration offset $y$ satisfies the following:

(i) $y$ is persistently exciting (PE), i.e., there exist $\mu_{0}>0$ and $T_{0}>0$ such that

$$
\int_{t}^{t+T_{0}} y(\varsigma)^{2} d \varsigma \geq \mu_{0}, \quad \forall t \geq 0 ;
$$

(ii) y crosses zero only at isolated points, i.e., for all $t_{k} \geq 0$ such that $y\left(t_{k}\right)=0$,

$$
\frac{d y}{d t}\left(t_{k}\right) \neq 0
$$

and, moreover, any two such points are separated by an interval of length no smaller than $T_{D}>0$, i.e., for all $t_{k}, t_{l} \geq 0$, such that $y\left(t_{k}\right)=0$ and $y\left(t_{l}\right)=0$ with $t_{k} \neq t_{l}$,

$$
\left|t_{k}-t_{l}\right| \geq T_{D}
$$

The hypothesis that $y$ is $\mathrm{PE}$ guarantees that $\tau(t)$ does not tend to a constant as $t \rightarrow \infty$. Thus, the time-scale transformation (17) is well posed. Finally, the conditions (23) and (24) guarantee that the switching signal $\rho(t):=\operatorname{sgn}(y(t))$ has a minimal dwell-time $T_{D}$. This, and the continuity of $\nu$ in (17), implies that $\sigma(\tau)$ also has a minimal dwell-time $\tau_{D}$.

We are now ready to present our first statement, for the case in which the parameters are known.

Proposition 1. Consider the system (12) with $K(t, y)$ given by (13), (20), and

$$
k_{1}^{+}>0, \quad k_{2}^{+}<0, \quad k_{1}^{-}=-k_{1}^{+}<0, \quad k_{2}^{-}=k_{2}^{+}<0 .
$$

If $v_{x}$ satisfies (4) and Assumption 1 holds, for the system (12), the origin $\{\tilde{w}=0\}$ is globally asymptotically stable.

Proof. The proof is inspired by [10] and [30]. The conditions $k_{1}^{+}>0, k_{2}^{+}<0, k_{1}^{-}<0, k_{2}^{-}<0$ ensure that the matrices $A_{+}, A_{-}$are Hurwitz, and a direct computation shows that the pairs $\left(A_{+}, C\right),\left(A_{-}, C\right)$ are observable. Hence, there exist two symmetric positive-definite matrices $P_{+}, P_{-}$such that $A_{+}^{\top} P_{+}+P_{+} A_{+}=-C^{\top} C$ and $A_{-}^{\top} P_{-}+P_{-} A_{+}=-C^{\top} C$ are satisfied [31, Prop. 5.4]. Furthermore, a direct computation shows that by imposing $k_{1}^{-}=-k_{1}^{+}, k_{2}^{-}=k_{2}^{+}$, the solutions of the above Lyapunov equations satisfy $P_{+}=P_{-}=: P$. Thus, $P$ defines a nonstrict (because $C^{\top} C$ is positive semidefinite) common Lyapunov function for the switched system (21). Under these conditions, and since the switching signal $\sigma(\tau)$ has a minimal dwell time $\tau_{D}$, it follows from [32, Th. 4] that the origin for the system (21) is globally exponentially stable, uniformly with respect to the switching signal. Thus, in the 


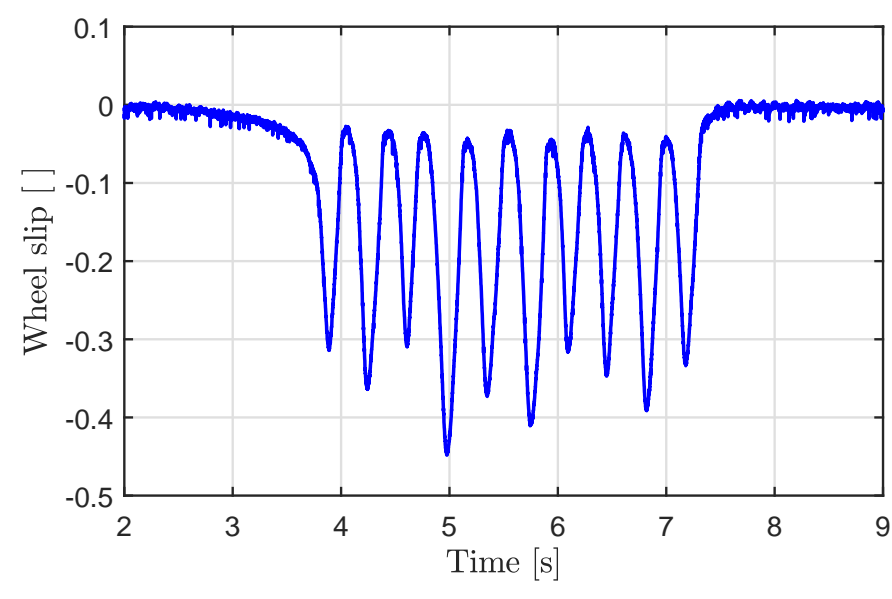

Fig. 2. Wheel slip measured during an experiment at $65 \mathrm{~km} / \mathrm{h}$. A five-phase hybrid ABS algorithm is active between $t=3.85 \mathrm{~s}$ and $t=7.27 \mathrm{~s}$.

original time-scale, the origin for the system (12) is globally asymptotically stable.

\section{Analysis under unknown road conditions}

Proposition 2. Consider the system (8) and the observer given by (10), (13), (20), and (25). Assume that $v_{x}$ satisfies (4), Assumption 1 holds, and $\Psi(t, u(t), y(t))$ is PE. Then, the origin $(\tilde{w}, \tilde{\theta})=(0,0)$ of $(11)$ is globally asymptotically stable.

Proof. The proof is inspired by Zhang [16]. Define the linear combination of the estimation errors $\eta:=\tilde{w}-\Upsilon \tilde{\theta}$. From (11b) and the definition of $\eta$, one has

$$
\dot{\tilde{\theta}}=-\Gamma \Upsilon^{\top} C^{\top} C[\Upsilon \tilde{\theta}+\eta] .
$$

Differentiating $\eta$ and using (11a), (11b) and (10c) one obtains

$$
\dot{\eta}=[A(t, y)-K(t, y) C] \eta \text {. }
$$

Note that the system formed by (26) and (27) has a cascade structure. Moreover, note that the dynamics of $\eta$ given by (27) has exactly the same form as that of the system (12). Thus, it follows from Proposition 1 that the origin of (27) is globally asymptotically stable. Now, consider the unforced system

$$
\dot{\tilde{\theta}}=-\Gamma \Upsilon^{\top} C^{\top} C \Upsilon \tilde{\theta}
$$

As $\Psi(t, u(t), y(t))$ is bounded and PE, $\Upsilon(t, u(t), y(t))$ governed by (10c) is also bounded and PE [33]. Hence, the origin of (28) is globally exponentially stable and (26) is input-to-state stable with respect to the input $\eta$. Moreover, the interconnection term $-\Gamma \Upsilon^{\top} C^{\top} C$ is bounded. It follows that the origin $(\tilde{\theta}, \eta)=(0,0)$ of the cascade $(26)-(27)$ is globally asymptotically stable [34]. Finally, to see that the same property holds for the origin $(\tilde{w}, \tilde{\theta})=(0,0)$ of $(11)$, we observe that $\tilde{w}=\eta+\Upsilon \tilde{\theta}$.

\section{EXPERIMENTAL RESULTS}

The performance of the proposed observer has been tested on experimental data acquired from the tyre-in-the-loop test rig of the Delft University of Technology during the evaluation of ABS strategies [35]. The test rig consists of a tyre rolling
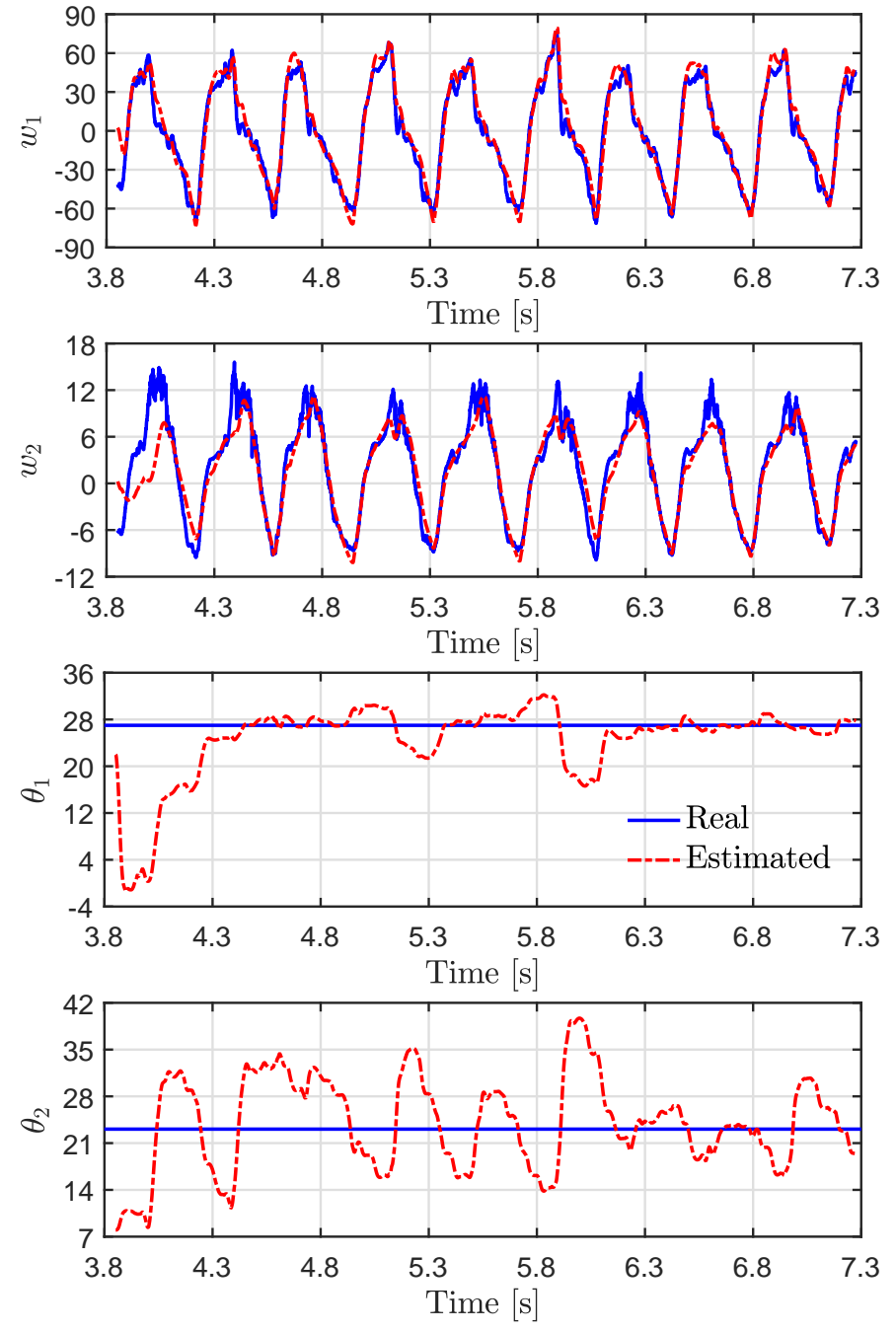

Fig. 3. Real vs estimated states and parameters of the system (8).

on top of a $2.5 \mathrm{~m}$ diameter steel drum (see [36, Fig. 12.7]). The tyre is mounted on a rim attached to a rotating axle that is, in turn, supported by a fixed frame through mechanical bearings. To emulate the displacement of the vehicle with respect to the road, the drum rotates about its axis driven by an electric motor. The braking of the wheel is performed via a hydraulic disk brake mounted on one side of the axle. The forces acting on the tyre are measured via piezoelectric force transducers, while the wheel and the drum speeds are measured by encoders.

Fig. 2 shows the wheel slip of an experiment [35] conducted at a drum speed of approximately $65 \mathrm{~km} / \mathrm{h}$. A five-phase hybrid ABS control algorithm [37] that modulates the brake pressure based on measurements of the wheel acceleration offset is active between $t=3.85 \mathrm{~s}$ and $t=7.27 \mathrm{~s}$; thus the switched adaptive observer is tested during that interval. To validate the estimation results, the data of Fig. 1 was fitted to the friction model (5) in order to identify the coefficients $c_{i}$. This allows to compute the XBS offline by evaluating the derivative of the fitted curve over the wheel slip. Then, the states and parameters of the system (8) can be computed offline from the wheel-acceleration-offset measurements and the parameters of 


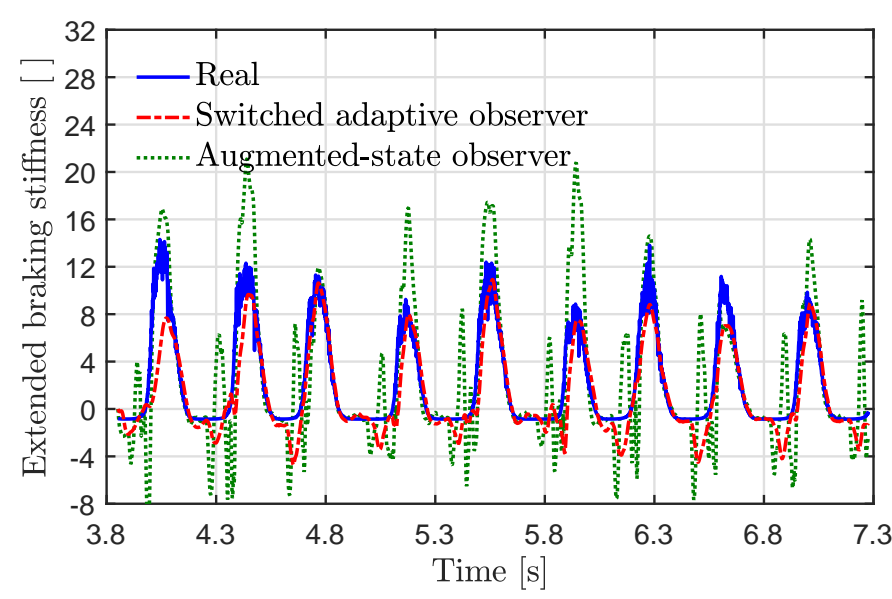

Fig. 4. Real vs estimated XBS. The switched adaptive observer exhibits an overall better performance than the augmented-state observer of [10].

the fitted friction curve.

The estimation results are illustrated in Fig. 3. The estimates of the states $w_{1}$ and $w_{2}$ converge to their real values within $0.5 \mathrm{~s}$, even though the estimates of the parameters $\theta_{1}$ and $\theta_{2}$ do not converge to a constant value. It should be noted, however, that the estimated parameters do approach their real values and remain close to them during the rest of the experiment. As a result, a good estimation of the XBS is obtained, as illustrated in Fig. 4. The results obtained via the augmented-state observer proposed in [10] are shown as well. The switched adaptive observer clearly exhibits an overall better performance.

\section{Simulation Results: TWO-AXLE VEHICLE MODEL}

In this section we present simulation results using a two-axle vehicle model [38]. The objective is to test the performance of the observer under changes in the road conditions (a perturbation that cannot be implemented in the test rig) and in the presence of vehicle dynamics that were not considered during the design stage. These include the suspension dynamics, as well as load transfer phenomenon and tyre relaxation length. Moreover, the simulations consider that the vehicle speed is estimated from the measurements of the angular velocities of the wheels and the longitudinal acceleration of the vehicle.

Figs. 5 to 7 illustrate a hard-braking scenario of a vehicle traveling at an initial speed of $180 \mathrm{~km} / \mathrm{h}$. The vehicle starts braking at $t=0.25 \mathrm{~s}$. The braking on each wheel is individually controlled by a six-phase ABS control algorithm that is activated at $t=0.75 \mathrm{~s}$ in the front axle, and at $t=0.50$ $\mathrm{s}$ in the rear axle. Changes in the road conditions occur at times $2.5 \mathrm{~s}$ and $5 \mathrm{~s}$ : during the first part of the simulation the vehicle runs on dry asphalt, then on snow, and then on wet asphalt. The vehicle speed at the end of the simulation is $15 \mathrm{~km} / \mathrm{h}$.

As with the experimental tests, the XBS estimation is performed during the interval in which the ABS is active. To evaluate the observer under realistic conditions, the vehicle speed is not considered to be directly measured. Instead, it is estimated using the Kalman-filter approach of [39], which takes as inputs the measurements of the linear velocity of the

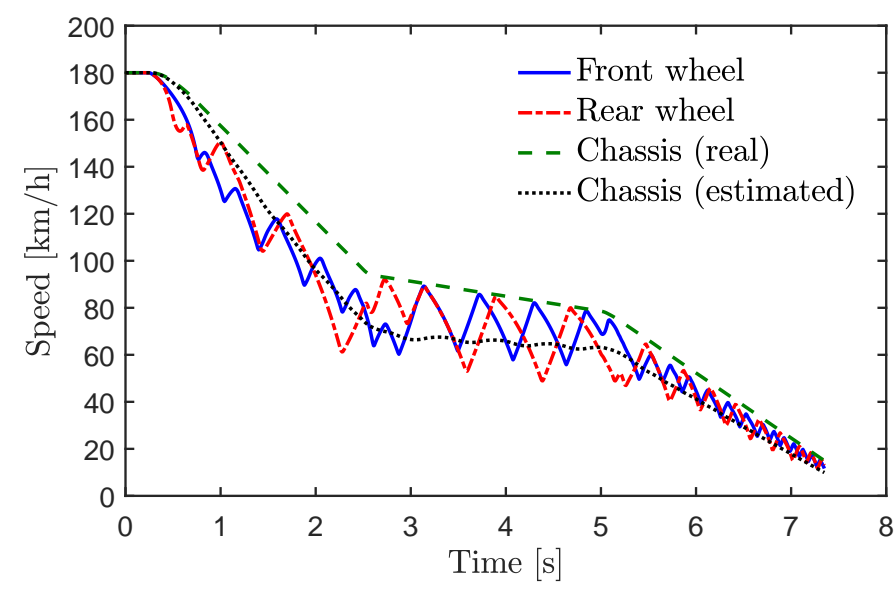

Fig. 5. Longitudinal speed of the vehicle and linear speed of the tyres at the wheel-ground contact point during an ABS braking scenario.

front wheel (computed as $R \omega$ ) and the longitudinal acceleration $a_{x}(t)$. The latter is affected by an additive dynamic bias with a turn-on component of $0.5 \mathrm{~m} / \mathrm{s}^{2}$, which is common in modern accelerometers [40]. The estimate of $v_{x}(t)$ used in the implementation of the observer is shown in Fig. 5. Because of the harsh braking conditions, the estimate displays an average error of $18 \%$ relative to its true value.

Moreover, in order to take into account the load transfer phenomenon in the implementation of (10), the parameter $a$ that depends on the vertical load on each wheel is computed online using the measurements of the vehicle longitudinal acceleration. That is, the vertical loads $F_{z_{f}}$ and $F_{z_{r}}$ in the front and rear wheels are computed as [13, § 2.4]

$$
\begin{aligned}
& F_{z_{f}}=W_{f}-\Delta_{F_{z}} a_{x}(t), \\
& F_{z_{r}}=W_{r}+\Delta_{F_{z}} a_{x}(t),
\end{aligned}
$$

where $W_{f}$ and $W_{r}$ are the static vertical loads at the front and rear wheels, and $\Delta_{F_{z}}$ is the coefficient of load transfer due to the vehicle acceleration. These three parameters are constant and depend on (known) geometrical and mechanical characteristics of the vehicle. Thus, the above expressions can be computed with the measurements of $a_{x}(t)$. Fig. 7 shows the vertical load on each wheel during the considered braking scenario. The dotted lines correspond to the values of $F_{z_{f}}$ and $F_{z_{r}}$ computed using (29). Even though the measurements of $a_{x}(t)$ are subject to the accelerometer's bias, the computed values follow closely the real values of the vertical load in each wheel, thus they provide a reasonable approximation for the parameter $a$ to be used in the observer.

The observer's robustness to the uncertainties described above is illustrated in Fig. 8. The graphic shows a comparison between the XBS estimated using the true signals $v_{x}(t)$ and $a_{x}(t)$ and that estimated using the vehicle's estimated speed (Fig. 5) and measured acceleration subject to sensor bias. Note in the latter case that even though the vehicle speed is underestimated by $18 \%$, the observer still provides a reasonable approximation of the XBS. Notably, the zerocrossing of the estimate coincides with that of the real XBS. This is important because the observer properly detects when the system transitions from one region of the tyre to the other. 


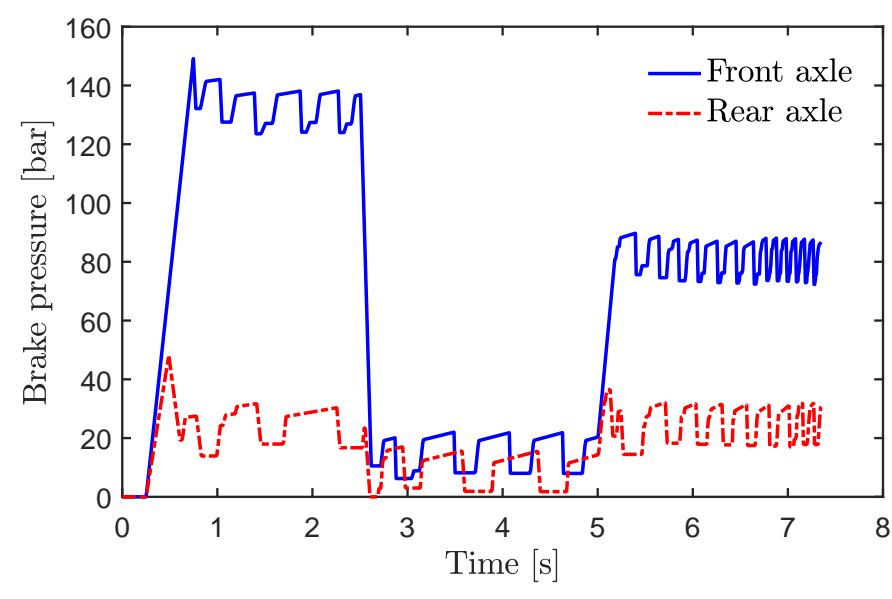

Fig. 6. Brake pressure in the front and rear wheels during an ABS braking scenario. The pressure is drastically reduced between $t=2.5 \mathrm{~s}$ and $t=5 \mathrm{~s}$ when the vehicle runs on a low-friction surface (snow).

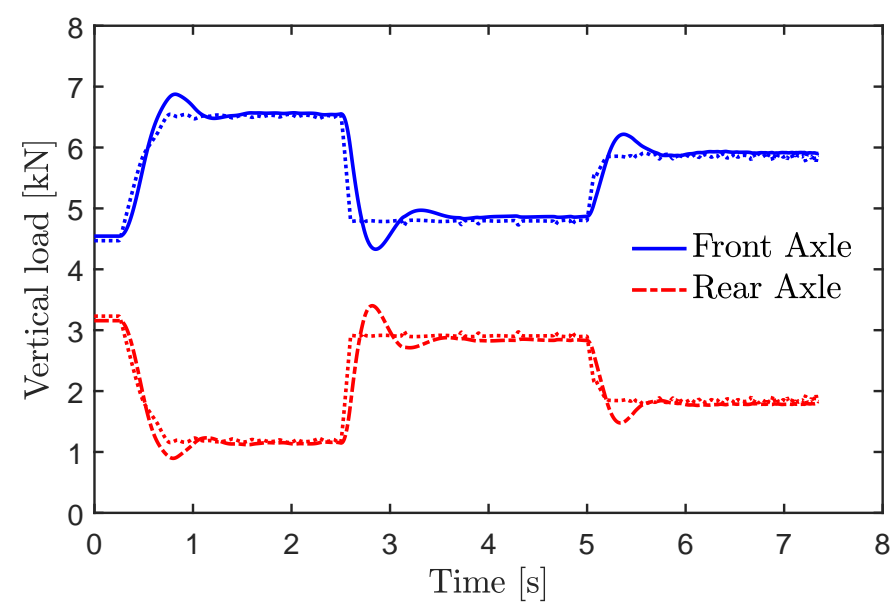

Fig. 7. Vertical load in the front and rear axles during an ABS braking scenario. The dotted lines correspond to the values of $F_{z_{f}}$ and $F_{z_{r}}$ computed using (29) that are used in the implementation of the observer.

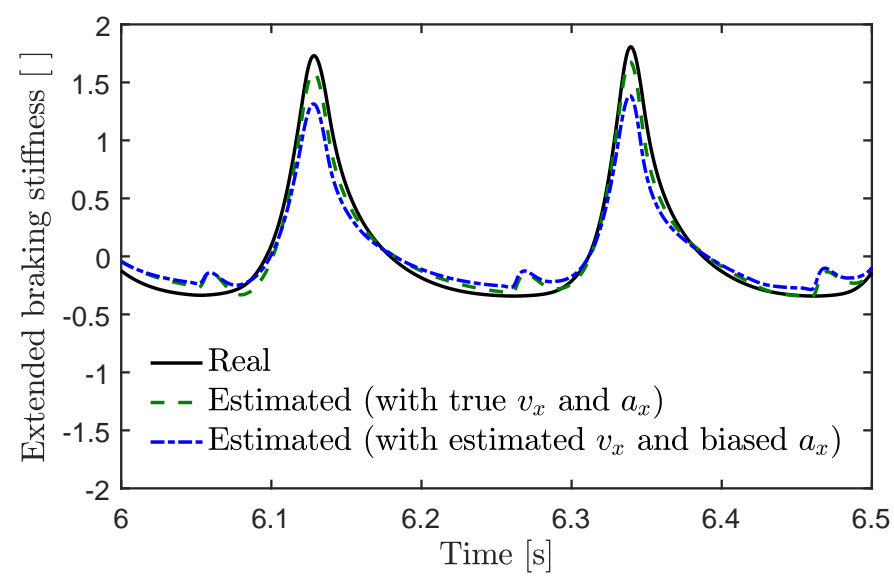

Fig. 8. Real vs estimated XBS of the front wheel. Comparison of the results obtained using the true signals $v_{x}(t)$ and $a_{x}(t)$ against those obtained using the estimated vehicle speed and the acceleration perturbed by measurement bias.

The observer's overall performance (i.e., considering all the aforementioned uncertainties and perturbations) is illustrated in Fig. 9. During the initial transient, the estimate of the front tyre exhibits a better performance than that of the rear tyre. Because the abrupt changes in the road conditions (and thus in the parameters $c$ and $d$ of the model (8)) cause a sudden growth of the error $y-C \hat{w}$ between the measured and the expected output in (10), the estimates are highly perturbed after the road transitions. However, they are able to converge to their true values within $0.5 \mathrm{~s}$ after the perturbation has occurred.

\section{CONCLUSiON}

This paper presented a switched adaptive observer for the estimation of the tyre XBS. The design is based on a simplified dynamic model that retains a good degree of accuracy during an ABS-controlled braking scenario. The performance of the observer has been tested in a tyre-in-the-loop test rig as well as via numerical simulations with a two-axle vehicle model with satisfactory results. Moreover, the observer is able to recover a good estimate of the XBS even after large changes in road conditions and can be easily implemented in a real vehicle equipped with an ABS without the need of additional sensors.

The approach presented here has however an important limitation. Because of the conditions of Assumption 1 related to the PE and zero-crossing transversality of the measured output, the XBS observer might not be used, for instance, in conjunction with a controller that aims at stabilizing the trajectories of the system (7) at the origin. It is possible, nevertheless, to exploit the XBS observer to improve the performance of ABS using hybrid control algorithms, is such a way that Assumption 1 be trivially satisfied. Work in this direction is currently under way. Additional future work will also consider the effects of combined slip and lateral forces on the estimation results.

\section{REFERENCES}

[1] C. Ahn, H. Peng, and H. E. Tseng, "Robust estimation of road frictional coefficient," IEEE Transactions on Control Systems Technology, vol. 21, no. 1, pp. 1-13, Jan. 2013.

[2] S. Müller, M. Uchanski, and K. Hedrick, "Estimation of the maximum tire-road friction coefficient," Journal of Dynamic Systems, Measurement, and Control, vol. 125, no. 4, pp. 607-617, Dec. 2003.

[3] R. Rajamani, N. Piyabongkarn, J. Lew, K. Yi, and G. Phanomchoeng, "Tire-road friction-coefficient estimation," IEEE Control Systems, vol. 30, no. 4, pp. 54-69, Aug. 2010.

[4] C. R. Carlson and J. C. Gerdes, "Consistent nonlinear estimation of longitudinal tire stiffness and effective radius," IEEE Transactions on Control Systems Technology, vol. 13, no. 6, pp. 1010-1020, Nov. 2005.

[5] F. Gustafsson, "Slip-based tire-road friction estimation," Automatica, vol. 33, no. 6, pp. 1087-1099, 1997.

[6] M. Sugai, H. Yamaguchi, M. Miyashita, T. Umeno, and K. Asano, "New control technique for maximizing braking force on antilock braking system," Vehicle System Dynamics, vol. 32, no. 4-5, pp. 299-312, 1999.

[7] T. Umeno, "Estimation of tire-road friction by tire rotational vibration model," R\&D Review of Toyota CRDL, vol. 37, no. 3, 2002.

[8] E. Ono, K. Asano, M. Sugai, S. Ito, M. Yamamoto, M. Sawada, and Y. Yasui, "Estimation of automotive tire force characteristics using wheel velocity," Control Engineering Practice, vol. 11, no. 12, pp. 1361-1370, 2003.

[9] J. Villagra, B. d'Andréa Novel, M. Fliess, and H. Mounier, "A diagnosisbased approach for tire-road forces and maximum friction estimation," Control Engineering Practice, vol. 19, no. 2, pp. 174-184, 2011.

[10] T. B. Hoàng, W. Pasillas-Lépine, A. De Bernardinis, and M. Netto, "Extended braking stiffness estimation based on a switched observer, with an application to wheel-acceleration control," IEEE Transactions on Control Systems Technology, vol. 22, no. 6, pp. 2384-2392, Nov. 2014. 


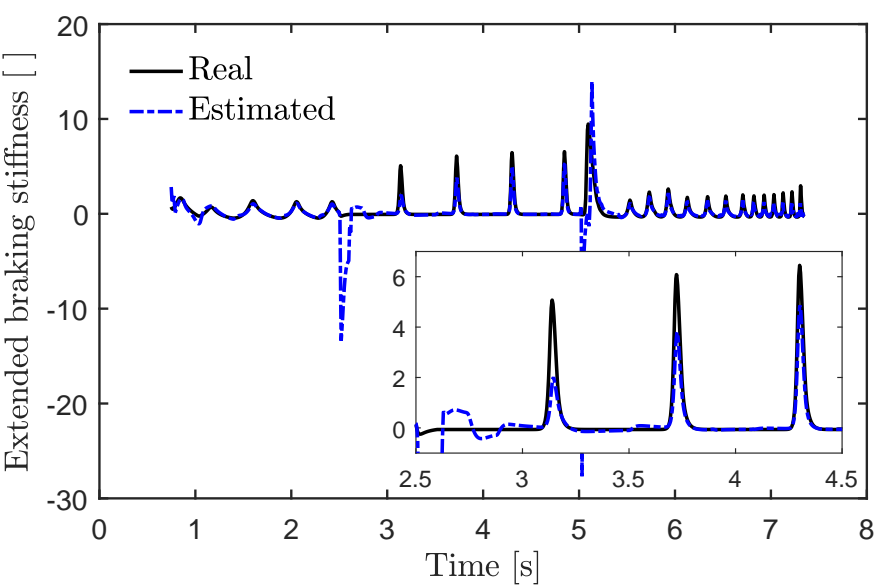

(a) Front axle

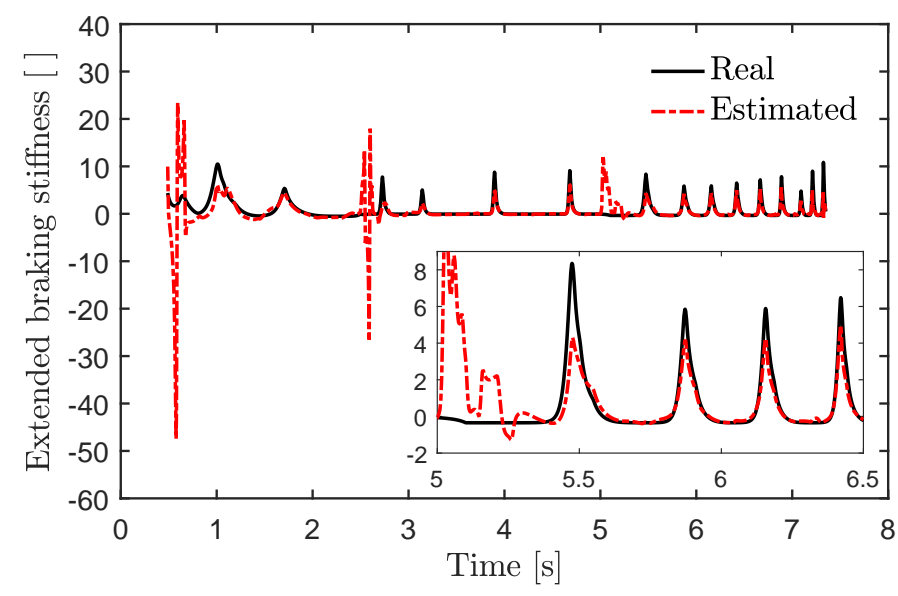

(b) Rear axle

Fig. 9. Real vs estimated XBS of the front and rear wheels. The estimates are highly perturbed after the abrupt changes in road conditions at $t=2.5 \mathrm{~s}$ and $t=5 \mathrm{~s}$, but they are able to re-approach their true values within $0.5 \mathrm{~s}$ after the perturbations.

[11] M. Burckhardt, Fahrwerktechnik: Radschlupf-Regelsysteme. Würzburg, Germany: Vogel-Verlag, 1993.

[12] M. Aguado-Rojas, W. Pasillas-Lépine, and A. Loría, "A switched adaptive observer for extended braking stiffness estimation," in 2018 American Control Conference (ACC), Jun. 2018, pp. 6323-6328.

[13] S. M. Savaresi and M. Tanelli, Active braking control systems design for vehicles, ser. Advances in Industrial Control. London, UK: SpringerVerlag, 2010.

[14] G. Besançon, "A viewpoint on observability and observer design for nonlinear systems," in New Directions in Nonlinear Observer Design, ser. Lecture Notes in Control and Information Sciences, H. Nijmeijer and T. Fossen, Eds. London, UK: Springer-Verlag, 1999, vol. 244, pp. 3-22.

[15] R. Marino and P. Tomei, Nonlinear Control Design: Geometric, Adaptive, and Robust. London, UK: Prentice Hall, 1995.

[16] Q. Zhang, "Adaptive observer for multiple-input-multiple-output (MIMO) linear time-varying systems," IEEE Transactions on Automatic Control, vol. 47, no. 3, pp. 525-529, Mar. 2002.

[17] _ _, "An adaptive observer for sensor fault estimation in linear time varying systems," IFAC Proceedings Volumes, vol. 38, no. 1, pp. 137142, 2005, 16th IFAC World Congress.

[18] X. Li, Q. Zhang, and H. Su, "An adaptive observer for joint estimation of states and parameters in both state and output equations," International Journal of Adaptive Control and Signal Processing, vol. 25, no. 9, pp. 831-842, 2011.

[19] A. Loría and E. Panteley, "Uniform exponential stability of linear timevarying systems: revisited," Systems \& Control Letters, vol. 47, no. 1, pp. 13-24, 2002.

[20] H. K. Khalil, Nonlinear Systems, 2nd ed. New Jersey, USA: Prentice Hall, 1996.

[21] V. V. Nemytskii and V. V. Stepanov, Qualitative Theory of Differential Equations. New Jersey, USA: Princeton University Press, 1960.

[22] M. Sampei and K. Furuta, "On time scaling for nonlinear systems: Application to linearization," IEEE Transactions on Automatic Control, vol. 31, no. 5, pp. 459-462, May 1986.

[23] L. Hsu, R. Ortega, and G. Damm, "A globally convergent frequency estimator," IEEE Transactions on Automatic Control, vol. 44, no. 4, pp. 698-713, Apr. 1999.

[24] M. Guay, "Observer linearization by output-dependent time-scale transformations," IEEE Transactions on Automatic Control, vol. 47, no. 10 pp. $1730-1735$, Oct. 2002.

[25] W. Respondek, A. Pogromsky, and H. Nijmeijer, "Time scaling for observer design with linearizable error dynamics," Automatica, vol. 40, no. 2, pp. 277-285, 2004.

[26] M. Aguado-Rojas, "On control and estimation problems in antilock braking systems," Ph.D. dissertation, Université Paris-Saclay, 2019.

[27] D. Liberzon, Switching in Systems and Control, ser. Systems \& Control: Foundations \& Applications. Boston, USA: Birkhäuser Basel, 2003.

[28] K. Reif, Ed., Brakes, brake control and driver assistance systems: Function, regulation and components, ser. Bosch Professional Automotive Information. Wiesbaden, Germany: Springer Vieweg, 2014.
[29] G. Mastinu and M. Ploechl, Eds., Road and off-road vehicle system dynamics handbook. Boca Raton, FL, USA: CRC Press, 2014.

[30] T. B. Hoàng, W. Pasillas-Lépine, and W. Respondek, "A switching observer for systems with linearizable error dynamics via singular time-scaling," in Proceedings of the 21st International Symposium on Mathematical Theory of Networks and Systems, 2014, pp. 1101-1108.

[31] W. J. Terrell, Stability and Stabilization: An Introduction. New Jersey, USA: Princeton University Press, 2009.

[32] J. P. Hespanha, "Uniform stability of switched linear systems: extensions of LaSalle's invariance principle," IEEE Transactions on Automatic Control, vol. 49, no. 4, pp. 470-482, Apr. 2004

[33] E. Panteley, A. Loría, and A. Teel, "Relaxed persistency of excitation for uniform asymptotic stability," IEEE Transactions on Automatic Control, vol. 46, no. 12, pp. 1874-1886, Dec. 2001.

[34] A. Loría and E. Panteley, "Cascaded nonlinear time-varying systems: Analysis and design," in Advanced Topics in Control Systems Theory: Lecture Notes from FAP 2004, ser. Lecture Notes in Control and Information Sciences, F. Lamnabhi-Lagarrigue, A. Loría, and E. Panteley, Eds. London, UK: Springer-Verlag, 2005, vol. 311, pp. 23-64.

[35] M. Gerard, W. Pasillas-Lépine, E. de Vries, and M. Verhaegen, "Improvements to a five-phase ABS algorithm for experimental validation," Vehicle System Dynamics, vol. 50, no. 10, pp. 1585-1611, 2012.

[36] H. B. Pacejka, Tire and Vehicle Dynamics, 3rd ed. Oxford, UK: Butterworth-Heinemann, 2012.

[37] W. Pasillas-Lépine, "Hybrid modeling and limit cycle analysis for a class of five-phase anti-lock brake algorithms," Vehicle System Dynamics, vol. 44 , no. 2 , pp. 173-188, 2006.

[38] G. Genta, Motor vehicle dynamics: modeling and simulation, ser. Advances in Mathematics for Applied Sciences. Singapore: World Scientific, 2006, vol. 43.

[39] M. Corno, G. Panzani, and S. M. Savaresi, "Traction-control-oriented state estimation for motorcycles," IEEE Transactions on Control Systems Technology, vol. 21, no. 6, pp. 2400-2407, Nov. 2013.

[40] V. Rodrigo Marco, J. Kalkkuhl, and J. Raisch, "EKF for simultaneous vehicle motion estimation and IMU bias calibration with observabilitybased adaptation," in 2018 American Control Conference (ACC), Jun. 2018, pp. 6309-6316. 